\section{Prescrição de antimicrobianos em unidades de saúde da família no Sul do Brasil}

\author{
Antimicrobial prescription in family health units \\ in Southern Brazil
}

\author{
1 Programa de Pós-graduação \\ em Ciências da Saúde, \\ Universidade Federal do \\ Rio Grande, \\ Rio Grande, Brasil. \\ 2 Centro de Ciências da \\ Saúde, Universidade da \\ Região da Campanha. \\ Bagé, Brasil. \\ 3 Programa de Pós-graduação \\ em Saúde Coletiva \\ Universidade do Vale do Rio \\ dos Sinos, São Leopoldo, \\ Brasil. \\ Correspondência \\ N. U. L. Tavares \\ Curso de Farmácia, Centro \\ de Ciências da Saúde, \\ Universidade da Região \\ da Campanha. \\ Av. Tupi Silveira 2099, Bagé, \\ RS 96400-000, Brasil. \\ noemiatavares@terra.com.br
}

\section{Abstract}

This paper describes antimicrobial prescription, clinical indications, and seasonal characteristics in primary health care. We performed a cross-sectional study in family health units in Bagé, Rio Grande do Sul State, Brazil, in July 2005 and January 2006. All medical prescriptions ( $n=2,877$ ) were analyzed, and the prevalence rates for antimicrobial prescription in winter and summer were 30.4\% (95\%CI: 28.5-32.4) and 21\% (95\%CI: 19.1-22.9), respectively. The most frequently prescribed antimicrobials were amoxicillin and sulfamethoxazole-trimethoprim. The main clinical indications for antimicrobial prescription were non-specific upper respiratory tract infections (22.5\%), throat infections (20.8\%), urinary tract infections (13.3\%), otitis (8.5\%), and sinusitis (7.5\%). We observed differences between summer and winter in prescriptions for the same diseases. Seasonal differences between treatment profiles for the same diseases show the absence of a consistent antimicrobial prescription pattern in primary health care, contrary to World Health Organization guidelines, which advocate the establishment of protocols for antimicrobial use at all levels of care, as a strategy to prevent inadequate usage and the occurrence of strains that are resistant to available antimicrobials.

Anti-Bacterial Agents; Drug Prescriptions; Primary Health Care
Noemia U. L. Tavares 1,2

Andréa D. Bertoldi 3

Ana Luiza Muccillo-Baisch 1

\section{Introdução}

Vários fatores influenciam a prescrição inadequada dos antimicrobianos, tais como a falta de conhecimento e expectativas, tanto dos prescritores como dos pacientes, fatores econômicos e culturais, características dos sistemas de saúde dos países e regulamentação ambiental ${ }^{1}$. O uso abusivo e indiscriminado desses medicamentos pode levar à emergência e aumento da resistência microbiana ${ }^{2}$.

O fenômeno da resistência microbiana é complexo e refere-se a cepas de microorganismos que são capazes de multiplicar-se em presença de concentrações de antimicrobianos mais altas do que as que provêm das doses terapêuticas dadas a humanos. É um fenômeno biológico natural que se seguiu à introdução de agentes antimicrobianos na prática clínica e as suas taxas variam na dependência do consumo local de antimicrobianos 3 . O seu crescimento acarreta dificuldades no manejo de infecções e contribui para o aumento dos custos do sistema de saúde, pois com a ineficácia dos antimicrobianos essenciais são necessários tratamentos com novos antimicrobianos, geralmente mais caros 4 .

A Organização Mundial da Saúde (OMS) preconiza, como uso apropriado de antimicrobianos, o uso eficaz em relação ao custo com o qual se obtém o máximo de efeito terapêutico com o mínimo de toxicidade e de potencial de desenvolvimento de resistência microbiana 1 . 
O uso inapropriado de antimicrobianos é um problema mundial. Para preveni-lo, são necessárias estratégias de controle em todos os níveis de cuidado e na maioria dos países para que o seu efeito total seja significativo. Diversos estudos têm documentado o uso inadequado de antimicrobianos em situações às quais estes não se aplicam, principalmente nas infecções de origem viral 5,6,7,8,9.

É fundamental, para a promoção do seu uso racional, tanto na atenção individual quanto na saúde pública, atentar para boas práticas de seleção e prescrição destes medicamentos 1. Estudos de prescrições são uma das estratégias para monitorização do uso de medicamentos e dos hábitos de prescrição. Têm sido descritos erros de prescrição de antimicrobianos que vão desde a indicação não apropriada para infecção a erros técnicos relacionados à duração do tratamento, dosagem, intervalo entre doses e via de administração incorretos 2,10.

Diante desta crescente preocupação com uso racional de antimicrobianos, a OMS e outros grupos, como Management Sciences for Health (MSH), desenvolveram indicadores de prescrição que permitem conhecer as práticas terapêuticas correntes e comparar parâmetros entre instituições similares. Vários estudos nacionais destes indicadores foram publicados nos últimos anos $11,12,13,14,15,16$

Outro elemento que afeta a prescrição e a utilização adequada dos medicamentos é a forma como estão organizadas as intervenções no processo saúde-doença. Segundo a literatura, a organização da atenção básica é uma tendência mundial. No Brasil, a Estratégia Saúde da Família, tem sido valorizada como um dos eixos centrais para a organização da atenção básica nos municípios. A Estratégia Saúde da Família tem como principal propósito reorganizar a prática da atenção à saúde em novas bases e substituir o modelo tradicional. Prioriza as ações de prevenção, promoção e recuperação da saúde das pessoas, de forma integral e contínua. Possibilita, portanto, uma compreensão ampliada do processo saúdedoença e da necessidade de intervenções, além das práticas curativas 17 .

Neste contexto, o presente estudo tem por objetivo descrever o perfil de prescrição de antimicrobianos, bem como as suas principais indicações clínicas, nas unidades básicas de saúde da família, em município da Região Sul do Brasil, avaliando suas diferenças sazonais. Com isto, espera-se contribuir para que futuras intervenções possam ser conduzidas na promoção do uso racional dos antimicrobianos.

\section{Métodos}

O estudo foi realizado nas unidades básicas de saúde da família da cidade de Bagé, Rio Grande do Sul, localizada na Região Sul do Brasil, que possui uma população de 120.129 habitantes, segundo dados do Censo Demográfico 2000 (Instituto Brasileiro de Geografia e Estatística, http:/ / www.ibge.gov.br). A estrutura da rede assistencial, no período do estudo, era composta por oito unidades básicas de saúde, com 13 equipes da Estratégia Saúde da Família. O total de famílias cadastradas na Estratégia Saúde da Família, no início do estudo, segundo dados da Secretaria Municipal de Saúde e Meio Ambiente, era de 11.053 famílias correspondendo a 41.529 pessoas, sendo que uma unidade ainda estava em fase de implantação e não estava com o cadastramento completo. A abrangência da Estratégia Saúde da Família no município era de $41 \%$ da população.

A coleta de dados foi realizada nos meses de julho (inverno) de 2005 e janeiro (verão) de 2006. O critério de inclusão das unidades de saúde no estudo foi ter no mínimo seis meses de implantação, para evitar problemas de estrutura assistencial e de representatividade da área adscrita por cadastramento incompleto. Das oito unidades, foram estudadas cinco que contavam com oito equipes de saúde da família. Duas unidades foram excluídas por terem menos de seis meses de implantação (três equipes da Estratégia Saúde da Família) e uma não apresentou fonte adequada de informação para que fossem coletados os dados de prescrição (duas equipes da Estratégia Saúde da Família).

Foi realizado um estudo transversal descritivo, onde foram analisadas todas as prescrições médicas oriundas das unidades básicas de saúde da família selecionadas, nos meses de julho de 2005 e janeiro de 2006, buscando informações sobre a prescrição de antimicrobianos e observando as diferenças sazonais da prescrição desses medicamentos no inverno e verão.

Foram incluídas prescrições de antimicrobianos constantes ou não na lista básica de padronização do município para uso sistêmico, excluindo-se as apresentações de uso tópico.

As variáveis independentes coletadas foram: idade agrupada posteriormente por faixas etárias classificando criança (0-9 anos), adolescente (1019 anos), adulto (20-59 anos) e idoso (60 anos ou mais); tipo de antimicrobiano prescrito; duração do tratamento e indicação clínica da prescrição. O instrumento para a coleta de dados foi uma planilha composta por uma parte específica sobre a prescrição de antimicrobianos e outra incluindo a idade dos pacientes. 
Os dados foram coletados a partir da ficha de atendimento médico dos pacientes atendidos, sendo que nos casos de presença de prescrição de antimicrobianos foram pesquisados, no prontuário do paciente, os dados de indicação clínica da prescrição. Após, os dados foram lançados em um banco de dados no programa Microsoft Office Excel 2003 (Microsoft Corp., Estados Unidos) e transferidos para o pacote estatístico Stata 8.0 (Stata Corp., College Station, Estados Unidos), onde foram realizadas as análises. A análise descritiva caracterizou o perfil das prescrições de antimicrobianos de forma geral, por grupos de idade e indicações clínicas, nos dois períodos estudados. Foi considerada, para o cálculo da prevalência de prescrição, a proporção de consultas em que se prescreveu pelo menos um antimicrobiano sistêmico. Realizou-se ainda uma estratificação por período sendo utilizado o teste do quiquadrado para as comparações de proporções nos dois períodos.

Os medicamentos foram classificados segundo a classificação Anatomical Therapeutic Chemical Classification (ATC) 18 recomendada pelo Drug Utilization Research Group da OMS para estudos de utilização de medicamentos, e as indicações clínicas segundo a Classificação Internacional de Doenças, 10a revisão (CID-10) 19.

O protocolo do estudo foi aprovado pelo Comitê de Ética em Pesquisa na Área da Saúde da Universidade Federal do Rio Grande. A Secretaria Municipal de Saúde e Meio Ambiente apoiou a realização do estudo após ser informada sobre os objetivos da pesquisa e que as informações obtidas através da coleta dos dados seriam utilizadas única e exclusivamente para a execução do presente estudo, e estas divulgadas de forma anônima.

\section{Resultados}

Durante os meses de julho de 2005 e janeiro de 2006, foram realizados 4.110 atendimentos para consultas médicas nas unidades básicas de saúde da família estudadas, sendo a prevalência de prescrição de antimicrobianos (proporção de consultas em que se prescreveu pelo menos um antimicrobiano sistêmico), nesses meses, de $30,4 \%$ (intervalo de 95\% de confiança - IC95\%: 28,5-32,4) e $21 \%$ (IC95\%: 19,1-22,9), respectivamente $(\mathrm{p}<0,001)$.

O total de atendimentos médicos nos dois períodos resultou em 2.877 prescrições médicas (70\% dos atendimentos). Todas as prescrições foram analisadas, contendo 8.242 medicamentos, o que corresponde a uma média de 2,9 medicamentos por prescrição, sendo 1.106 desses, antimicrobianos de uso sistêmico. Do total de antimicrobianos sistêmicos, $62,8 \%$ foram prescritos no mês referente ao inverno e $37,2 \%$, no referente ao verão.

A lista básica de antimicrobianos padronizados no município não sofreu alterações nos dois períodos de estudo e não houve descontinuidade no abastecimento dos mesmos. Os antimicrobianos não padronizados prescritos foram: azitromicina, doxiciclina, ciprofloxacino, fenoximetilpenicilina e norfloxacino. Observou-se que houve mudança do prescritor em quatro das equipes de saúde da família das unidades estudadas no mês do verão. As temperaturas médias na cidade de Bagé, em julho 2005 e janeiro de 2006, foram, respectivamente, de $12,7^{\circ} \mathrm{C}$ e $24,2^{\circ} \mathrm{C}$, segundo informação do Instituto Nacional de Meteorologia (INMET).

A Tabela 1 demonstra a distribuição dos antimicrobianos prescritos por faixas etárias durante o inverno. Nesse período, a proporção de antimicrobianos sistêmicos prescritos no total de medicamentos foi de $14,2 \%$.

A amoxicilina foi o antimicrobiano mais prescrito, correspondendo a cerca de metade das prescrições de antimicrobianos por faixa etária e a associação de sulfametoxazol/trimetropin foi o segundo, representando quase um quarto das prescrições em todas as faixas etárias. A cefalexina representou $8 \%$ do total de prescrições de antimicrobianos nesse período, sendo mais prescrita entre os adultos e idosos, e o metronidazol, $6 \%$ do total das prescrições, sendo mais freqüente sua prescrição para adolescentes e adultos jovens. Os demais antimicrobianos apresentaram proporções de prescrição inferiores a $5 \%$ (Tabela 1).

A Tabela 2 indica a mesma distribuição, porém, no período do verão, onde a proporção de prescrição de antimicrobianos sistêmicos no total de medicamentos foi de 11,9\%. Apesar de ser o antimicrobiano mais prescrito, a amoxicilina apresentou um menor percentual de prescrição em relação ao inverno ( $\mathrm{p}<0,001)$, representando cerca de $30 \%$ das prescrições em todas as faixas etárias. A associação de sulfametoxazol/ trimetropin continuou sendo o segundo antimicrobiano mais prescrito com cerca de $20 \%$ das prescrições, sendo estas menos freqüentes nos grupos mais jovens (0-19 anos) em relação aos adultos e idosos $(p=0,049)$. A eritromicina foi o terceiro antimicrobiano mais prescrito no verão, sendo tão prescrito quanto a amoxicilina no grupo de $0-9$ anos (cerca de $30 \%$ das prescrições desta faixa etária). A cefalexina também apresentou um aumento na proporção de prescrições em relação ao período do inverno $(8,2 \%$ para $13,6 \%$ ), sendo o segundo antimicrobiano 
Antimicrobianos sistêmicos prescritos em julho de 2005 por faixa etária nas unidades básicas de saúde da família. Bagé, Rio Grande do Sul, Brasil.

\begin{tabular}{|c|c|c|c|c|c|}
\hline \multirow[t]{3}{*}{ Antimicrobianos * } & \multicolumn{5}{|c|}{ Faixa etária (anos completos) } \\
\hline & $0-9$ & $10-19$ & $20-59$ & 60 ou mais & Todos \\
\hline & $n(\%)$ & n (\%) & n (\%) & $\mathrm{n}(\%)$ & $n(\%)$ \\
\hline Amoxicilina & $161(55,9)$ & $65(46,7)$ & $90(40,0)$ & $23(53,4)$ & $339(48,7)$ \\
\hline Ampicilina & $0(0,0)$ & $4(2,8)$ & $2(0,8)$ & $0(0,0)$ & $6(0,8)$ \\
\hline Azitromicina & $0(0,0)$ & $0(0,0)$ & $1(0,4)$ & $0(0,0)$ & $1(0,1)$ \\
\hline Cefalexina & $22(7,6)$ & $7(5,0)$ & $23(10,2)$ & $5(11,6)$ & $57(8,2)$ \\
\hline Ciprofloxacino & $0(0,0)$ & $0(0,0)$ & $3(1,3)$ & $0(0,0)$ & $3(0,4)$ \\
\hline Doxiciclina & $0(0,0)$ & $1(0,7)$ & $2(0,8)$ & $0(0,0)$ & $3(0,4)$ \\
\hline Eritromicina & $4(1,3)$ & $2(1,4)$ & $3(1,3)$ & $0(0,0)$ & $9(1,2)$ \\
\hline Fenoximetilpenicilina & $0(0,0)$ & $0(0,0)$ & $1(0,4)$ & $0(0,0)$ & $1(0,1)$ \\
\hline Metronidazol & $5(1,7)$ & $14(10,0)$ & $22(9,7)$ & $2(4,6)$ & $43(6,1)$ \\
\hline Norfloxacino & $0(0,0)$ & $2(1,4)$ & $2(0,8)$ & $0(0,0)$ & $4(0,5)$ \\
\hline Penicilina benzatina & $11(3,8)$ & $11(7,9)$ & $9(4,0)$ & $2(4,6)$ & $33(4,7)$ \\
\hline Penicilina procaína & $3(1,0)$ & $1(0,7)$ & $4(1,7)$ & $0(0,0)$ & $8(1,1)$ \\
\hline Sulfatometoxazol/Trimetropin & $82(28,4)$ & $32(23,0)$ & $63(28,0)$ & $11(25,5)$ & $188(27,0)$ \\
\hline Total & $288(100,0)$ & $139(100,0)$ & $225(100,0)$ & $43(100,0)$ & $695(100,0)$ \\
\hline
\end{tabular}

* Classificados pela Anatomical Therapeutic Chemical Classification (ATC) 18

Antimicrobianos sistêmicos prescritos em janeiro de 2006 por faixa etária nas unidades básicas de saúde da família. Bagé, Rio Grande do Sul, Brasil.

\begin{tabular}{|c|c|c|c|c|c|}
\hline \multirow[t]{3}{*}{ Antimicrobianos * } & \multicolumn{5}{|c|}{ Faixa etária (anos completos) } \\
\hline & $0-9$ & $10-19$ & $20-59$ & 60 ou mais & Todos \\
\hline & $n(\%)$ & n (\%) & $n(\%)$ & n (\%) & n (\%) \\
\hline Amoxicilina & $48(31,3)$ & $26(36,1)$ & $36(25,5)$ & $17(37,7)$ & $127(30,9)$ \\
\hline Ampicilina & $0(0,0)$ & $1(1,3)$ & $4(2,8)$ & $2(4,4)$ & $7(1,7)$ \\
\hline Cefalexina & $16(10,4)$ & $15(20,8)$ & $21(14,8)$ & $4(8,8)$ & $56(13,6)$ \\
\hline Doxiciclina & $0(0,0)$ & $0(0,0)$ & $0(0,0)$ & $1(2,2)$ & $1(0,2)$ \\
\hline Eritromicina & $45(29,4)$ & $10(13,8)$ & $11(7,8)$ & $3(6,6)$ & $69(16,7)$ \\
\hline Fenoximetilpenicilina & $0(0,0)$ & $0(0,0)$ & $1(0,7)$ & $0(0,0)$ & $1(0,2)$ \\
\hline Metronidazol & $8(5,2)$ & $3(4,1)$ & $12(8,5)$ & $2(4,4)$ & $25(6,0)$ \\
\hline Norfloxacino & $0(0,0)$ & $1(1,3)$ & $9(6,3)$ & $1(2,2)$ & $11(2,6)$ \\
\hline Penicilina benzatina & $10(6,5)$ & $4(5,5)$ & $6(4,2)$ & $4(8,8)$ & $24(5,8)$ \\
\hline Penicilina procaína & $0(0,0)$ & $0(0,0)$ & $4(2,8)$ & $1(2,2)$ & $5(1,2)$ \\
\hline Sulfatometoxazol/Trimetropin & $26(16,9)$ & $12(16,6)$ & $36(25,5)$ & $10(22,2)$ & $84(20,4)$ \\
\hline Tetraciclina & $0(0,0)$ & $0(0,00)$ & $1(0,71)$ & $0(0,00)$ & $1(0,24)$ \\
\hline Total & $153(100,0)$ & $72(100,0)$ & $141(100,0)$ & $45(100,0)$ & $411(100,0)$ \\
\hline
\end{tabular}

* Classificados pela Anatomical Therapeutic Chemical Classification (ATC) 18.

mais prescrito na faixa dos 10-19 anos de idade $(\mathrm{p}=0,004)$.

As principais indicações clínicas para prescrição de antimicrobianos, nos dois períodos, foram as seguintes: infecção das vias aéreas superiores não especificadas (22,5\%), amigdalites $(20,8 \%)$, infecções do trato urinário $(13,3 \%)$, otites $(8,5 \%)$ e sinusites (7,5\%). Também foram prescritos vin- te tratamentos com antimicrobianos para gripes e resfriados. Verificou-se que em $19,4 \%$ dos prontuários não constava a informação da indicação clínica para a prescrição e 3,8\% dos prontuários não foram localizados.

A Tabela 3 demonstra a distribuição dos antimicrobianos prescritos para essas indicações clínicas no período do inverno. No tratamento 
das infecções das vias aéreas superiores, não especificadas, a amoxicilina foi o antimicrobiano mais prescrito representando mais da metade das prescrições. O sulfametoxazol/trimetropin foi o segundo com $24,3 \%$ de prescrição, seguido da cefalexina com $8,1 \%$.

Para o tratamento das amigdalites, a amoxicilina também representou metade das prescrições e a penicilina benzatina foi prescrita em um terço dos casos. Nas infecções do trato urinário $41,8 \%$ das prescrições foram de sulfametoxazol/ trimetropin sendo a amoxicilina e a cefalexina os segundos e terceiros antimicrobianos mais prescritos com cerca de $20 \%$ das prescrições cada um. Para o tratamento das otites e sinusites, cerca de $80 \%$ das prescrições foram de amoxicilina, e o sulfametoxazol/trimetropin foi o segundo antimicrobiano mais prescrito (Tabela 3 ).

A Tabela 4 indica a distribuição dos antimicrobianos utilizados nas principais indicações clínicas no período do verão. Nas infecções das vias aéreas superiores, não especificadas, a amoxicilina também foi o antimicrobiano mais prescrito, porém apresentou uma diminuição ( $\mathrm{p}=$ $0,008)$ do percentual de prescrição em relação ao inverno (de $64,2 \%$ para $41,9 \%$ ). O sulfameto$\mathrm{xazol} /$ trimetropin continuou sendo o segundo antimicrobiano mais prescrito com cerca de $20 \%$ das prescrições. A eritromicina e a penicilina benzatina, que praticamente não foram prescritas no inverno, representaram $11,6 \%$ e $7 \%$ das prescrições no verão, respectivamente.

Nas amigdalites, a eritromicina teve um aumento significativo em relação ao inverno ( $\mathrm{p}<$ 0,001 ), sendo o antimicrobiano mais prescrito (42,9\% das prescrições). A amoxicilina sofreu uma redução no percentual de prescrição para cerca de $37 \%(p=0,02)$, e a penicilina benzatina foi o terceiro antimicrobiano mais prescrito (16,3\%). Nas infecções do trato urinário, o sulfametoxazol/trimetropin, assim como no inverno, foi o antimicrobiano mais prescrito. Houve uma diminuição da proporção de prescrição da amoxicilina para $10,9 \%$, apesar de não significativa $(\mathrm{p}=0,1)$, e da cefalexina para $6,5 \%(\mathrm{p}=0,04)$ e um aumento na prescrição do norfloxacino (de 4,5\% para $21,7 \%)$, para o tratamento das mesmas ( $\mathrm{p}=$ 0,005) (Tabela 4).

O perfil de prescrição para as otites e sinusites, que no inverno foi bastante semelhante, se diferenciou no verão. Verificou-se que a prescrição da amoxicilina diminuiu para cerca de um terço ( $\mathrm{p}<0,001$ ), e que o sulfametoxazol/trimetropin foi o antimicrobiano mais prescrito para as otites, enquanto que para as sinusites, este apresentou um percentual de prescrição igual ao

Distribuição dos antimicrobianos sistêmicos prescritos para as cinco principais indicações clínicas segundo a Classificação Internacional de Doenças, 10 ạ revisão (CID-10) 19 em julho de 2005 nas unidades básicas de saúde da família. Bagé, Rio Grande do Sul, Brasil.

\begin{tabular}{|c|c|c|c|c|c|c|c|}
\hline Antimicrobianos * & $\begin{array}{c}\text { Infecção das } \\
\text { vias aéreas } \\
\text { superiores não } \\
\text { especificadas } \\
\text { n (\%) }\end{array}$ & Amigdalite & $\begin{array}{c}\text { Infecção } \\
\text { do trato } \\
\text { urinário } \\
\text { n (\%) }\end{array}$ & n (\%) & Sinusite & Outras & n (\%) \\
\hline Amoxicilina & $95(64,2)$ & $43(54,4)$ & $15(22,3)$ & $37(78,7)$ & $32(80,0)$ & $41(28,3)$ & $263(50,0)$ \\
\hline Ampicilina & $0(0,0)$ & $0(0,0)$ & $3(4,5)$ & $0(0,0)$ & $0(0,0)$ & $2(1,4)$ & $5(0,95)$ \\
\hline Azitromicina & $1(0,7)$ & $0(0,0)$ & $0(0,0)$ & $0(0,0)$ & $0(0,0)$ & $0(0,0)$ & $1(0,2)$ \\
\hline Cefalexina & $12(8,1)$ & $2(2,5)$ & $14(20,9)$ & $2(4,3)$ & $3(7,5)$ & $2(1,4)$ & $35(6,6)$ \\
\hline Ciprofloxacino & $0(0,0)$ & $0(0,0)$ & $3(4,5)$ & $0(0,0)$ & $0(0,0)$ & $0(0,0)$ & $3(0,6)$ \\
\hline Doxiciclina & $0(0,0)$ & $0(0,0)$ & $0(0,0)$ & $0(0,0)$ & $0(0,0)$ & $2(1,4)$ & $2(0,4)$ \\
\hline Eritromicina & $1(0,7)$ & $2(2,5)$ & $0(0,0)$ & $0(0,0)$ & $0(0,0)$ & $3(2,0)$ & $6(1,1)$ \\
\hline Fenoximetilpenicilina & $1(0,7)$ & $0(0,0)$ & $0(0,0)$ & $0(0,0)$ & $0(0,0)$ & $0(0,0)$ & $1(0,2)$ \\
\hline Metronidazol & $0(0,0)$ & $0(0,0)$ & $0(0,0)$ & $0(0,0)$ & $0(0,0)$ & $36(24,8)$ & $36(6,8)$ \\
\hline Norfloxacino & $0(0,0)$ & $0(0,0)$ & $3(4,5)$ & $0(0,0)$ & $0(0,0)$ & $0(0,0)$ & $4(0,8)$ \\
\hline Penicilina benzatina & $0(0,0)$ & $26(32,9)$ & $1(1,5)$ & $1(2,1)$ & $0(0,0)$ & $5(3,5)$ & $33(6,3)$ \\
\hline Penicilina procaína & $2(1,3)$ & $2(2,5)$ & $0(0,0)$ & $1(2,1)$ & $0(0,0)$ & $2(1,4)$ & $7(1,3)$ \\
\hline Sulfatometoxazol/Trimetropin & $36(24,3)$ & $4(5,0)$ & $28(41,8)$ & $6(12,8)$ & $5(12,5)$ & $51(35,2)$ & $130(24,7)$ \\
\hline Total & $148(100,0)$ & $79(100,0)$ & $67(100,0)$ & $47(100,0)$ & $40(100,0)$ & $145(100,0)$ & $526(100,0)$ \\
\hline
\end{tabular}

* Classificados pela Anatomical Therapeutic Chemical Classification (ATC) 18. 
Distribuição dos antimicrobianos sistêmicos prescritos para as cinco principais indicações clínicas segundo a Classificação Internacional de Doenças, 10a revisão (CID-10) 19 em janeiro de 2006 nas unidades básicas de saúde da família. Bagé, Rio Grande do Sul, Brasil.

\begin{tabular}{|c|c|c|c|c|c|c|c|}
\hline Antimicrobianos * & $\begin{array}{c}\text { Infecção das } \\
\text { vias aéreas } \\
\text { superiores não } \\
\text { especificadas } \\
\text { n (\%) }\end{array}$ & Amigdalite & $\begin{array}{c}\text { Infecção } \\
\text { do trato } \\
\text { urinário } \\
\text { n (\%) }\end{array}$ & n (\%) & Sinusite & n (\%) & n (\%) \\
\hline Amoxicilina & $18(41,9)$ & $36(36,7)$ & $5(10,9)$ & $9(36,0)$ & $8(33,3)$ & $13(14,9)$ & $89(27,5)$ \\
\hline Ampicilina & $1(2,3)$ & $1(1,0)$ & $2(4,3)$ & $0(0,0)$ & $1(4,1)$ & $1(1,1)$ & $6(1,9)$ \\
\hline Cefalexina & $4(9,3)$ & $1(1,0)$ & $3(6,5)$ & $4(16,0)$ & $4(16,7)$ & $24(27,6)$ & $40(12,4)$ \\
\hline Doxiciclina & $1(2,3)$ & $0(0,0)$ & $0(0,0)$ & $0(0,0)$ & $0(0,0)$ & $0(0,0)$ & $1(0,3)$ \\
\hline Eritromicina & $5(11,6)$ & $42(42,9)$ & $0(0,0)$ & $1(4,0)$ & $3(12,5)$ & $13(14,9)$ & $64(19,8)$ \\
\hline Fenoximetilpenicilina & $1(2,3)$ & $0(0,0)$ & $0(0,0)$ & $0(0,0)$ & $0(0,0)$ & $0(0,0)$ & $1(0,3)$ \\
\hline Metronidazol & $0(0,0)$ & $0(0,0)$ & $0(0,0)$ & $0(0,0)$ & $0(0,0)$ & $18(20,7)$ & $18(5,6)$ \\
\hline Norfloxacino & $0(0,0)$ & $0(0,0)$ & $10(21,7)$ & $0(0,0)$ & $0(0,0)$ & $1(1,1)$ & $11(3,4)$ \\
\hline Penicilina Benzatina & $3(7,0)$ & $16(16,3)$ & $0(0,0)$ & $0(0,0)$ & $0(0,0)$ & $2(2,3)$ & $21(6,50)$ \\
\hline Penicilina Procaína & $1(2,3)$ & $0(0,0)$ & $0(0,0)$ & $0(0,0)$ & $0(0,0)$ & $1(1,1)$ & $2(0,6)$ \\
\hline Sulfatometoxazol/Trimetropin & $9(20,9)$ & $2(2,0)$ & $26(56,5)$ & $11(44,0)$ & $8(33,3)$ & $14(16,1)$ & $70(21,7)$ \\
\hline Total & $43(100,0)$ & $98(100,0)$ & $46(100,0)$ & $25(100,0)$ & $24(100,0)$ & $87(100,0)$ & $323(100,0)$ \\
\hline
\end{tabular}

* Classificados pela Anatomical Therapeutic Chemical Classification (ATC) 18.

da amoxicilina (33,3\%). A cefalexina apresentou um aumento da sua prescrição $(p=0,04)$, ficando com cerca de $16 \%$ das prescrições, tanto nas otites quanto nas sinusites (Tabela 4 ).

Entre as demais indicações clínicas, os antimicrobianos mais prescritos foram o sulfametoxazol/trimetropin, no inverno, para tratamento de bronquite e a cefalexina, no verão, com cerca da metade das suas indicações para as infecções da pele. O metronidazol foi o antimicrobiano mais prescrito para as doenças sexualmente transmissíveis (DSTs) nos dois períodos.

A duração dos tratamentos prescritos foi em média de 7,6 dias (desvio-padrão-DP = 2,9), sendo que em 15,6\% das prescrições de antimicrobianos não foi encontrada esta informação.

\section{Discussão}

A OMS vem divulgando a importância e a necessidade de avaliação das condições de saúde e das práticas envolvendo o uso de medicamentos de maneira sistemática e padronizada 20 .

A literatura disponível apresenta um grande arsenal de indicadores de uso de medicamentos a partir de trabalhos do MSH e da OMS. Esses indicadores têm como intenção principal detectar pontos que devem ser questionados e reavaliados para desencadear ações corretoras subseqüen- tes. Entre eles estão os indicadores de prescrição que refletem o funcionamento dos prestadores de assistência à saúde em relação a vários aspectos-chave do uso racional de medicamentos 17.

Para a realização de estudos de prescrições que utilizam coleta de dados retrospectivos, é de extrema importância obter fontes adequadas de informação sobre as prescrições. Pode-se salientar a disponibilidade das cópias das prescrições na unidade de saúde e os prontuários de registro de história clínica dos pacientes 20.

Apesar de não ter sido possível coletar os dados de todas as unidades básicas de saúde da família, foi possível analisar todas as prescrições médicas das unidades incluídas na amostra nos dois períodos estudados. Salienta-se, também, o acesso aos prontuários dos pacientes, que possibilitou a correlação entre a prescrição e a indicação clínica para uso dos antimicrobianos. Entretanto, em cerca de $20 \%$ dos mesmos, não foi encontrado esse registro, impossibilitando a identificação da indicação clínica do antimicrobiano prescrito.

A porcentagem de consultas em que se prescreve pelo menos um antimicrobiano é um indicador da racionalidade da prescrição, tendo em vista que este grupo farmacológico é de alto custo e que a inadequação do seu uso pode acarretar prejuízos tanto em nível individual para o usuário do medicamento quanto para sociedade 
em que ele está inserido, em função da pressão seletiva exercida por todo o uso destes fármacos. No presente estudo, os valores encontrados para este indicador (30,4\% e $21 \%$ ) estão de acordo com o sugerido pela International Network for Rational Use of Drugs, que testou todos os indicadores básicos de uso de medicamentos em 12 países de renda média ou baixa e apontou valores entre $20 \%$ e $30 \%$ como aceitáveis para o indicador de prescrição de antimicrobianos 1,20.

Quando comparados com estudos nacionais realizados em unidades de saúde da família, observamos que os valores encontrados são semelhantes ao relatado por Farias et al. 16 (21,1\%) e superiores ao observado por Colombo et al. 12 (12,5\%). Em outros estudos, em unidades com modelo de atenção tradicional, os resultados para este indicador também foram semelhantes aos encontrados no presente estudo (20,9\%-26\%) 10,11,12,13,14,15,16, e em alguns países da África e da Ásia, os valores ficaram acima de $40 \% 20$. Apesar de apresentarem perfis epidemiológicos diferentes, a comparação desse indicador com resultados de outros estudos nacionais ou de outros países com modelos de atenção à saúde diferentes serve como parâmetro de avaliação da Estratégia Saúde da Família, no que se refere à atenção integral aos pacientes que passa pela racionalização das medidas terapêuticas, permitindo determinar os âmbitos prioritários em que se devam concentrar medidas futuras de intervenção 20 .

Considerando aspectos sazonais na prescrição de antimicrobianos, foram encontradas diferenças significativas entre as coletas de dados nos períodos do inverno e do verão sendo a maior proporção de prescrição no inverno. Este resultado era esperado, tendo em vista que o inverno é um período de temperaturas climáticas mais baixas, com um maior acometimento de doenças como as do trato respiratório, nas quais os antimicrobianos são largamente usados. Especialmente na região do estudo, as diferenças climáticas são bastante acentuadas, o que foi confirmado pelas diferentes médias de temperatura nos dois períodos. Em outro estudo realizado em Campo Grande, Mato Grosso do Sul 21, onde não existem diferenças climáticas tão expressivas, a prescrição de antimicrobianos também foi maior no inverno $(34,4 \%)$ do que no verão $(27,6 \%)$, sendo ambas superiores às do presente estudo.

O número de medicamentos prescritos, por receita, também é um indicador de prescrição e determina o grau de polimedicação a que a população pesquisada está sujeita 20 . Tendo em vista que no Brasil, segundo informações do Sistema Nacional de Informações Tóxico-Farmacológicas (SINITOX), os medicamentos ocupam o primeiro lugar entre os agentes causadores de intoxicações em seres humanos e o segundo lugar nos registros de mortes por intoxicação, e que apenas $50 \%$ dos pacientes, em média, tomam corretamente seus medicamentos, estes problemas são agravados quanto maior for o número de medicamentos utilizados 22 .

No presente estudo, encontraram-se, em média, 2,9 medicamentos por prescrição, valor este considerado alto pela OMS, que considera 1,3 a 2,2 medicamentos, por prescrição, um resultado sem tendência à polimedicação 20 . Em outros estudos nacionais $10,11,12,13,14,15,16$, todos os valores encontrados foram mais baixos (entre 1,5 e 2,3) e, também em estudo internacional, envolvendo 12 países de baixa e média renda, esses valores ficaram abaixo de 2,2, com exceção da Índia e Nigéria, que tiveram 3,3 e 3,8 medicamentos por prescrição, respectivamente 20 .

Considerando o número total de medicamentos prescritos, foi encontrada uma proporção de prescrição de antimicrobianos sistêmicos maior no inverno $(14,2 \%)$ do que no verão $(11,9 \%)$. Esses valores foram superiores aos de um estudo realizado em unidades básicas de saúde de município do norte do Paraná 15 , que encontrou 10,9\% de prescrição de antimicrobianos de uso sistêmico no total de medicamentos prescritos.

Das cinco principais indicações clínicas para a prescrição de antimicrobianos, quatro foram para tratamento de doenças do trato respiratório superior (amigdalites, infecção das vias aéreas superiores não especificadas, otites e sinusites) que segundo a literatura internacional representam as mais freqüentes razões de consulta médica e estão associadas a 75\% do total de prescrições de antimicrobianos 23 . Na sua maioria, estas são de etiologia viral, e de acordo com recomendações internacionais 5,6,7,8,24, o uso de antimicrobianos de rotina para o seu tratamento não é indicado, ficando restrito para pacientes com suspeita confirmada de infecção bacteriana e para profilaxia, quando as conseqüências da infecção possam ser severas. As otites e sinusites, mesmo em caso de diagnóstico de infecção bacteriana, poderiam ser tratadas por medidas desobstrutivas e de drenagem das secreções respiratórias 25 . Esse dado é preocupante, tendo em vista que a proposta da Estratégia Saúde da Família são as ações de promoção, proteção e recuperação da saúde familiar, de forma integral e contínua, onde o prescritor tem condições de prescrever antimicrobianos para estas patologias de forma mais criteriosa.

Entre as razões citadas pela literatura, para o uso de antimicrobianos nestas patologias, está a dificuldade da diferenciação da etiologia viral da bacteriana destas infecções. Como conseqüência do tratamento antimicrobiano das infecções de etiologia viral, pode correr a promoção de seleti- 
vidade e disseminação de resistência microbiana na comunidade 25,26,27.

As infecções do trato urinário têm a sua terapia antimicrobiana melhor conduzida pela descrição clínica onde a escolha do tratamento antimicrobiano vai depender da sua localização 28 . Recente estudo internacional mostrou que os germes mais presentes nestas infecções na comunidade são os bacilos gram-negativos, predominando a Escherichia coli com $67 \%$. Também foi verificada a sua sensibilidade frente a diversos antimicrobianos, encontrando-se $79 \%$ de sensibilidade ao sulfametoxazol/trimetropin 29, que foi o mais prescrito para o tratamento dessas infecções nos dois períodos do presente estudo.

A prescrição de medicamentos de acordo com a Lista Municipal de Padronização é considerada um indicador de aceitação da mesma pelos prescritores 18 que determina o grau de adequação das práticas de prescrição à Política Nacional de Medicamentos em função da tendência a prescrever a partir da relação local de medicamentos padronizados.

A recomendação, para esse indicador, é de que ele tenha valores altos. Entretanto, a avaliação desse indicador deve ser feita de maneira criteriosa, pois ele pode sugerir tanto uma seleção e padronização adequada, baseada no perfil epidemiológico de doenças da região, como pode refletir apenas o perfil de prescrição dos médicos da região mais do que o perfil de morbi-mortalidade 11 .

No presente estudo, $98,2 \%$ das prescrições exclusivamente de antimicrobianos no inverno e $96,8 \%$, no verão, estavam de acordo com a Lista Municipal de Padronização e apesar dos valores encontrados mostrarem uma alta aceitação da mesma pelos prescritores, vários antimicrobianos não padronizados foram prescritos. Entre eles, o norfloxacino foi o mais prescrito. É importante levar-se em consideração que a prescrição de medicamentos fora da lista padronizada do município pode levar ao insucesso terapêutico por falta de acesso ao medicamento 1 .

O presente estudo não se propõe a avaliar a adequação da indicação clínica para os antimicrobianos prescritos, mas convém ressaltar que vinte tratamentos antimicrobianos foram descritos pelos prescritores com a indicação "gripe/ resfriado", sendo que o uso destes fármacos em qualquer dos casos é considerado excessivo 30.

Quanto à duração do tratamento, observou-se que cerca de $15 \%$ das prescrições não continham esta informação, valor superior ao encontrado por Abrantes et al. 10 (10\%), que em estudo de avaliação da qualidade da prescrição de antimicrobianos, evidenciou a necessidade de imple- mentação de medidas informativas e normativas para a melhoria da qualidade das prescrições. A Política Nacional de Medicamentos enfatiza que a prescrição de medicamentos é o ato de definir o medicamento a ser consumido pelo paciente, com a respectiva dosagem e duração do tratamento 17. A falta desta informação na prescrição, especialmente se tratando de antimicrobianos, pode acarretar dúvidas e levar a falhas quanto ao cumprimento do tratamento. Salienta-se que o uso insuficiente dos antimicrobianos devido à falta de acesso, doses inadequadas, e não cumprimento de todo o tratamento, pode ser tão importante para a resistência bacteriana como o seu uso excessivo 1.

A OMS, preocupada com o aumento da resistência antimicrobiana, tem recomendado a elaboração de protocolos de uso de antimicrobianos em todos os níveis de cuidado ${ }^{1}$. Em seu guidelines há recomendações de abordagens de diferentes doenças infecciosas, muitas delas tratáveis na atenção básica 31,32 .

Além da utilização dos protocolos internacionais, como estratégia de uma política adequada da utilização de antimicrobianos, são recomendadas a elaboração de protocolos nacionais ou municipais e a criação de laboratórios de referência para que o uso de antimicrobianos seja baseado no conhecimento da morbi-mortalidade das infecções mais prevalentes e no perfil local de sensibilidade aos antimicrobianos 1,18.

Essa recomendação é de extrema importância quando se trata da atenção básica, que tem peculiaridades próprias e onde o controle do uso de antimicrobianos se diferencia de forma importante do uso hospitalar. Nos hospitais, existem comissões e especialistas sobre infecção hospitalar, listas de uso restrito e guias terapêuticos, e o médico prescreve conhecendo os dados sobre resistência bacteriana do hospital, além de não existir a automedicação e haver um controle sobre a evolução clínica e cumprimento do tratamento dos pacientes, o que não ocorre na atenção básica 33

A Espanha, que é um dos países desenvolvidos em que mais se consomem antimicrobianos, publicou recentemente um consenso sobre a utilização de antimicrobianos na atenção primária, elaborado por associações médicas e farmacêuticas que atuam na atenção primária no país. Esse documento descreve como o processo de uso de antimicrobianos deve ser conduzido pelos atores envolvidos: médicos, farmacêuticos e administradores sanitários, contribuindo para a diminuição do uso inadequado desses medicamentos 33 .

No presente estudo, houve uma mudança importante no perfil de prescrição de antimicro- 
bianos para o tratamento das mesmas patologias nos dois períodos estudados. Levando em consideração que ocorreram mudanças em metade dos profissionais prescritores de um período para outro, fica evidenciado que a prescrição destes medicamentos pode sofrer influências das condutas individuais de prescrição independente da sazonalidade.
Isso enfatiza a importância de recomendações oficiais para uso dessa classe terapêutica, na atenção básica, evitando o seu uso inapropriado e a modificação do perfil de sensibilidade aos antimicrobianos disponíveis na comunidade, preservando assim a eficácia do arsenal terapêutico disponível para o tratamento de doenças infecciosas.

\section{Resumo}

O artigo descreve o perfil das prescrições de antimicrobianos, suas principais indicações clínicas e aspectos sazonais. Realizou-se um estudo transversal nas unidades de saúde da família do Município de Bagé, Rio Grande do Sul, Brasil, nos meses de julho de 2005 e janeiro de 2006. Foram analisadas todas $(n=2.877)$ as prescrições médicas, e a prevalência de prescrição de antimicrobianos encontrada nos meses de inverno e verão foi de 30,4\% (IC95\%: 28,5-32,4) e 21\% (IC95\%: 19,1-22,9), respectivamente. Os antimicrobianos mais prescritos foram a amoxicilina e a associação de sulfametoxazol/trimetropin. As principais indicações clínicas para sua prescrição foram: infecção das vias aéreas superiores não especificadas (22,5\%), amigdalites (20,8\%) e infecções do trato urinário (13,3\%). Observou-se uma mudança no perfil de prescrição de antimicrobianos para o tratamento das mesmas patologias entre os dois períodos. Os resultados evidenciam a falta de um padrão de prescrição de antimicrobianos na atenção básica à saúde, o que contraria as recomendações da Organização Mundial da Saúde, que sugerem a criação de protocolos de uso de antimicrobianos em todos os níveis de cuidado, como estratégia para a prevenção do uso inadequado dos antimicrobianos disponíveis.

Agentes Antibacterianos; Prescrição de Medicamentos; Atenção Primária à Saúde

\section{Colaboradores}

N. U. L. Tavares escolheu o tema em estudo, conduziu o trabalho de campo, participou das análises e redação do manuscrito. A. D. Bertoldi realizou as análises, participou da redação do artigo e supervisão do trabalho como um todo. A. L. Muccillo-Baisch orientou todo o trabalho desde o seu projeto até a elaboração do artigo final.

\section{Referências}

1. World Health Organization. WHO global strategy for containment of antimicrobial resistance. Geneva: World Health Organization; 2001.

2. Fijn R, Chow MC, Schuur PM, De Jong-Van den Berg LT, Brouwers JR. Multicentre evaluation of prescribing concurrence with anti-infective guidelines: epidemiological assessment of indicators. Pharmacoepidemiol Drug Saf 2002; 11:361-72.

3. Wannmacher L. Uso indiscriminado de antibióticos e resistência microbiana: uma guerra perdida? Uso racional de medicamentos: temas selecionados. Brasília: Organização Pan-Americana da Saúde/Organização Mundial da Saúde; 2004.

4. World Health Organization. Using indicators to measure country pharmaceutical situations. Geneva: World Health Organization; 2006.

5. Gonzales R, Bartlett JG, Besser RE, Cooper RJ, Hickner JM, Hoffman JR, et al. Principles of appropriate antibiotic use for treatment of treatment of nonspecific upper respiratory tract infections in adults: background. Ann Intern Med 2001; 134:490-4.

6. Belongia EA, Naimi TS, Gale CM, Besser RE. Antibiotic use and upper respiratory infections: a survey of knowledge, attitudes, and experience in Wisconsin and Minnesota. Prev Med 2002; 34: 346-52.

7. Williams Jr. JW, Aguilar C, Cornell J, Chiquette E, Dolor RJ, Makela M, et al. Antibiotics for acute maxillary sinusitis. In: The Cochrane Library, Issue 4, 2003. Oxford: Update Software.

8. Del Mar CB, Glasziou PP, Spinks AB. Antibiotics for sore throat. In: The Cochrane Library, Issue 3, 1997. Oxford: Update Software.

9. Chung A, Perera R, Brueggemann AB, Elamin AE, Harnden A, Mayon-White R, et al. Effect of antibiotic prescribing on antibiotic resistance in individual children in primary care: prospective cohort study. BMJ 2007; 335:429. 
10. Abrantes PM, Magalhães SMS, Acurcio FA, Sakurai E. Avaliação da qualidade das prescrições de antimicrobianos dispensadas em unidades públicas de saúde de Belo Horizonte, Minas Gerais, Brasil, 2002. Cad Saúde Pública 2007; 23:95-104.

11. Santos V, Nitrini SO. Indicadores do uso de medicamentos prescritos e de assistência ao paciente de serviços de saúde. Rev Saúde Pública 2004; 38:819-26.

12. Colombo D, Helena ETS, Agostinho ACMG, Didjurgett JSMA. Padrão de prescrição de medicamentos nas unidades de Programa Saúde da Família de Blumenau. Rev Bras Ciênc Farm 2004; 40:549-58.

13. Acurcio FA, Perini E, Magalhães SMS, Vieira Terceiro LG, Coutinho KEO, Caldeira KV, et al. Analysis of medical prescriptions dispensed at health centers in Belo Horizonte, Minas Gerais, Brazil. Cad Saúde Pública 2004; 20:72-9.

14. Naves JOS, Silver LD. Evaluation of pharmaceutical assistance in public primary care in Brasília, Brazil. Rev Saúde Pública 2005; 39:223-30.

15. Silva PV, Girotto E. A prescrição de medicamentos em um município do norte do Paraná. Rev Bras Epidemiol 2006; 9:226-34.

16. Farias AD, Cardoso MAA, Medeiros ACD, Belém LF, Simões MOS. Prescription indicators in Family Health basic units of the municipal district of Campina Grande, PB. Rev Bras Epidemiol 2007; 10:149-56.

17. Marin N, Luiza VL, Osório-de-Castro CGS, Machado-dos-Santos S. Assistência farmacêutica para gerentes municipais. Rio de Janeiro: Organização Pan-Americana da Saúde/Organização Mundial da Saúde; 2003.

18. WHO Collaborating Centre for Drug Statistics Methodology. Anatomical therapeutic chemical (ATC) classification index with defined daily doses (DDDs). Oslo: World Health Organization; 2000.

19. Organização Mundial da Saúde. Classificação estatística internacional de doenças e problemas relacionados à saúde, 10a revisão. São Paulo: Centro Colaborador da OMS para a Classificação de Doenças em Português; 1995.

20. World Health Organization. How to investigate drug use in health facilities. Geneva: World Health Organization/Action Programme on Essential Drugs; 1993.
21. Cunha MCN, Zorzatto JR, Castro LLC. Avaliação do uso de medicamentos na rede pública municipal de saúde de Campo Grande, MS. Rev Bras Ciênc Farm 2002; 38:217-27.

22. Agência Nacional de Vigilância Sanitária. Parcerias para diminuir o mau uso de medicamentos. Rev Saúde Pública 2006; 40:191-2.

23. McCaig LF, Hughes JM. Trends in antimicrobial drug prescribing among office-based physicians in the United States. JAMA 1995; 273:214-9.

24. Browning GG. Childhood otalgia: acute otitis media. 1. Antibiotics not necessary in most cases. BMJ 1990; 300:1005-6.

25. Berquó LS, Barros AJD, Lima RC, Bertoldi AD. Utilização de antimicrobianos em uma população urbana. Rev Saúde Pública 2004; 38:239-46.

26. Pichichero ME. Understanding antibiotic overuse for respiratory tract infections in children. Pediatrics 1999; 104:1384-8.

27. Kristiansen BE, Sandnes RA, Mortensen L, Tveten Y, Vorland L. The prevalence of antibiotic resistance in bacterial respiratory pathogens from Norway is low. Clin Microbiol Infect 2001; 7:682-7.

28. Hooton TM, Stamm WE. Diagnosis and treatment of uncomplicated urinary tract infection. Infect Dis Clin North Am 1997; 11:551-81.

29. Ti TY, Kumarasinghe G, Taylor MB, Tan SL, Ee A, Chua C, Low A. What is true community-acquired urinary tract infection? Comparison of pathogens identified in urine from routine outpatient specimens and from community clinics in a prospective study. Eur J Clin Microbiol Infect Dis 2003; 22: 242-5.

30. Arrol B, Kenealy T. Antibiotics for the common cold and acute purulent rhinitis. In: The Cochrane Library, Issue 4, 1998. Oxford: Update Software.

31. Ministry of Health. Clinical practice guidelines: use of antibiotics in adults. Singapore: Ministry of Health; 2006.

32. Ministry of Health. Clinical practice guidelines: use antibiotics in pediatric care. Singapore: Ministry of Health; 2002

33. Vicente VB, González AB, Fadini ED, Vilena AE, Riera TE, Fernández JI, et al. Documento de consenso sobre la utilización de antibióticos en atención primaria. Aten Primaria 2006; 38:174-7.

Recebido em 25/Jul/2007

Versão final reapresentada em 11/Dez/2007 Aprovado em 26/Dez/2007 